\title{
NEREUS: engineering concept for an underwater mass spectrometer
}

\author{
Harry Hemond*, Richard Camilli \\ R.M. Parsons Laboratory, Department of Civil and Environmental Engineering, Massachusetts Institute of \\ Technology, Cambridge, MA 02139, USA
}

\begin{abstract}
NEREUS (novel, efficient, rapid evaluation of underwater spectra) is a self-contained underwater mass-spectrometer system capable of continuous measurements of gases and vapors dissolved in the water column. Rapid in-situ measurement minimizes or eliminates artefacts that are often a problem when collecting and storing samples for analysis of volatile constituents, and provides higher spatial and temporal resolution of chemical patterns than is possible with conventional techniques. NEREUS is designed to operate either independently on a mooring or winch line, or aboard an autonomous underwater vehicle (AUV), such as the Sea Grant Odyssey class submersible. Underwater mass spectrometers such as NEREUS have many important applications to practical problems, such as pollution monitoring, as well as to fundamental Earth systems research. (C) 2002 Published by Elsevier Science B.V. All rights reserved.
\end{abstract}

Keywords: AUV; Cycloidal; Mass spectrometer; Underwater

\section{Introduction}

Knowledge of the identity, concentration, and distribution of dissolved volatile substances is of great value in monitoring and managing water quality. Much fundamental research, ranging from identification of hidden hydrologic circulation patterns [1] and exploration of exotic marine ecosystems [2] to global climate modeling [3] and plate tectonic modeling based on

*Corresponding author. Tel.: +1-617-253-1637; Fax: +1-617258-8850. E-mail: hfhemond@mit.edu gases from deep sea hydrothermal vents [4], depends on measurement of dissolved trace gases. However, conventional measurement of volatile substances in water typically requires that samples be collected and taken to a laboratory for analysis, a process that is often slow and labor-intensive, and that requires precautions against chemical and physical changes occurring during collection and transport (e.g. degassing, photochemistry, microbial metabolism). Existing in-situ devices (e.g. dissolved oxygen (DO) probes) are commonly limited to detecting one or a few gas species [5], with separate sensors required for each species, and sensitivities of typically about $1 \mathrm{ppm}$; the latter can be an important limitation where sub-ppm concentrations of gases, such as $\mathrm{O}_{2}$, are ecologically significant. Continuous sampling techniques, popular among marine researchers (e.g. Weiss equilibrator), are generally limited to shipboard use and modest sampling depths, and have gas species-dependent equilibration times that range from minutes to hours [6,7]. As a result, the spatial and temporal resolution of data gathered by conventional methods is often lower than is needed to test hypotheses regarding certain environmental processes.

An in-situ, underwater mass spectrometer (MS) can overcome many of these limitations. One major advantage is the universality and the sensitivity of chemical measurement by mass spectrometry. An MS can yield mass spectra for virtually any gas or vapor that is admitted to its ion source, and the spectra can be quickly processed to determine both identity and concentration in mixtures of several compounds. Further, MSs can operate without reagents or 
exhaust, an important advantage in an underwater instrument, where discharge of any substance to the water column may be resisted by a large pressure difference and may also affect the buoyancy and trim of a platform vehicle. Further, in-situ measurement avoids the many artefacts that are possible when samples are collected and handled prior to analysis for volatile substances.

Although there are various means of interfacing an MS to the water column, the simplest and most satisfactory for many purposes is a membrane inlet. In this technique, a semipermeable membrane separates the sample from the high-vacuum system of the MS. Gases in the water diffuse through the membrane and enter the source region of the MS. At steady state, the analyte flux and thus the height of corresponding peaks in the mass spectrum are proportional to the gas concentration on the wet side of the membrane (e.g. [8]). Membrane permeability, which is the product of the solubility and the molecular diffusion coefficient of a gas in the membrane material, is thus a key factor in relating the flux of the gas to its aqueous concentration. Early use of membrane-inlet mass spectrometry for measurement of gases in laboratory settings is described by Hoch and Kok [9], while more recent discussions of membrane inlet MS in environmental contexts are provided by Kotiaho [10] and Richardson [11].

In practice, trade-offs must be made between inlet geometry and material, and the performance (speed of response, sensitivity) of a membrane-inlet MS. Thin membranes are favored for fast response and sensitivity [12], but membranes must also be thick enough to resist the pressure differential across them. Further, in the case of underwater membraneinlet MS, the water can vary greatly in temperature and pressure. Because devices to control temperature and pressure of the water contacting the membrane complicate inlet-system design, simple inlets for underwater MS may require empirical calibration both for changes in temperature (e.g. Maden and Hayward [12]) and for changes in water pressure (e.g. by physical deformation and possible permeability effects) in the case of deep-diving systems.

Mobile or field-portable membrane-inlet MSs share some of the attributes of underwater MS (i.e. need for small size and power consumption) and are in some respects precursors, despite the fact that many mobile MSs require vehicle transport and have power requirements in the hundreds of watts. Many mobile MSs have been intended for pollution studies (e.g. [13-15]). Miniature mass analyzers, as reviewed by Badman and Cooks [16], are clearly a desirable feature of any field system, although miniaturization of the mass analyzer does not itself assure a successful system, inasmuch as inlet, vacuum, data, and related peripheral systems also play a large part in determining overall size, weight, and performance. This is likely to be especially true of systems intended for use aboard small, low-cost underwater platforms, including many AUVs, where volume and weight constraints are severe, and batteries are the only power source.

Varying degrees of miniaturization and lowpower design appear in current underwater MS designs. Short et al. [17] describe an underwater MS system that uses either a quadrupole (Leybold Inficon Transpector) or ion-trap (Varian Saturn 2000) analyzer, and is carried aboard the Ocean Explorer AUV. The membrane inlet is described as a short $(10 \mathrm{~cm})$, polymer capillary through which thermostatted sample or calibration water is pumped. VOCs (volatile organic compounds), such as toluene, have been measured at the ppb level or lower using this system. Power consumption is of the order of 100 watts at 24 volts. McMurty and Smith [18] describe a submersible MS having the options of either a membrane or an electrospray inlet, and using a novel rotating-field MS. This is, to the authors' knowledge, the only application of electrospray, which can admit species of low volatility, to an underwater MS. A third underwater MS system is described by Matz et al. [19] and is based on a Bruker Franzen Model EM640 mobile MS; this instrument uses a hollow-fiber membrane inlet that alternately sorbs and desorbs analyte, then separates the des- 
orbed analyte on a gas chromatographic column. The instrument operates from a surface ship and is powered via its tether.

\section{NEREUS system description}

NEREUS (novel, efficient, rapid evaluation of underwater spectra) is a new underwater membrane-inlet MS system that offers significant advantages with respect to size, weight, power requirement and potential depth capability. The system is derived from a back-packable, batterypowered MS described by Hemond [20]. NEREUS is designed to operate aboard high-performance, low-cost Odyssey class AUVs, on a mooring, or on a winch line (Fig. 1). The instrument is optimized for measurement of low-molecular-weight gases and vapors, including many biogenic gases, permanent atmospheric gases, and light hydrocarbons, at levels down to tens of $\mathrm{ppb}$.

NEREUS fits within the upper hemisphere of a standard 17-inch (43-cm) diameter Benthos glass pressure sphere (rated for depths to 6000 $\mathrm{m})$. This avoids the cost of a customized pressure housing, and is also compatible with Odyssey class AUVs, which are designed around the same pressure spheres. The analyzer and electronics are designed specifically for low-

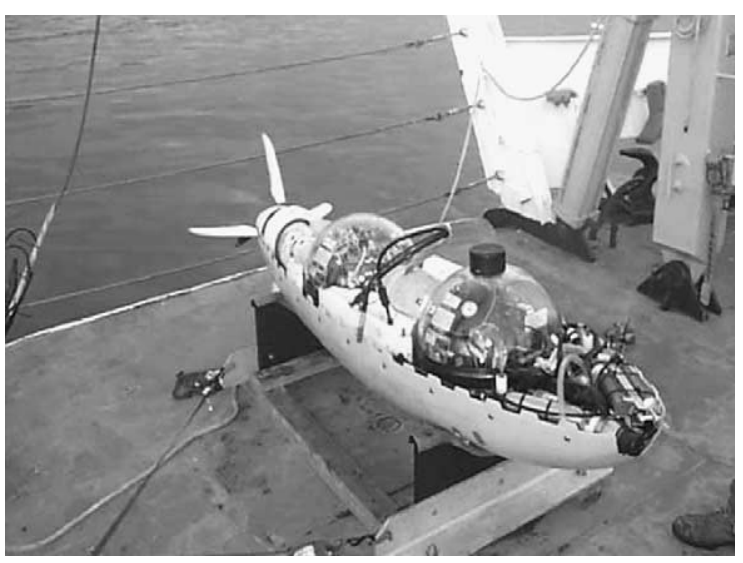

Fig. 1. Odyssey II autonomous underwater vehicle, with outer, free-flooding hull opened, exposing payload pressure spheres. NEREUS is contained within one such sphere (photo courtesy of MIT Sea Grant). power operation, using less than 20 watts in continuous operation and as low as 2 watts in "sleep" mode (filament and scanning circuitry off). Power is supplied from an internally housed battery, providing $12 \mathrm{~h}$ of operation, or may be drawn from a host AUV during extended missions. The analyzer and the vacuum system contain no moving parts or waste reservoirs, making the instrument insensitive to position and eliminating the need for active ballasting during long-term deployments. The NEREUS concept employs a simple, passive, flat-plate, membrane-inlet system, which conveys large depth capability.

The functional configuration of NEREUS is shown in Fig. 2. A membrane, exposed to the water column, allows analyte gas inflow to the MS while blocking bulk-liquid water flow. The MS includes an ion source, a cycloidal mass analyzer and a Faraday cup detector. A permanent magnet provides a homogeneous B-field for the mass analyzer, while an ion pump maintains the necessary high-vacuum environment in the analyzer. Analyte ions of a given $\mathrm{m} / \mathrm{z}$ are focused on the detector, and the current is amplified by an electrometer and recorded by a computerized data-acquisition system. The computer also calculates the potentials needed to scan across the desired $m / z$ range.

\subsection{Mass analyzer}

The analyzer used in NEREUS is of the same cycloidal design as used by Hemond [20] and described in further detail by Robinson [21]; its size advantage accrues from its inherent doublefocusing characteristic (Fig. 3). The ion path of the analyzer fits within a $2-\mathrm{cm}$ radius; yet Robinson was able to demonstrate a resolution in excess of 2500 using a similar analyzer with small modifications (a B-field of 0.69 tesla and narrowed slits). The relationship among $\mathrm{B}, \mathrm{E}$, and $m / z$ for a cycloidal analyzer is given by Bleakney and Hipple [22]. The $\mathrm{m} / \mathrm{z}$, range of NEREUS is approximately 12-150; for lower $\mathrm{m} / \mathrm{r}$, the analyzer can be operated as a $180^{\circ}$ sector instrument using a secondary Faraday 
cup collector, providing unit-mass resolution down to $m / z=2$.

\subsection{Circuit and magnet design}

NEREUS requires very little power for $\mathrm{m} / \mathrm{z}$ scanning, because only a near-static DC voltage is needed to determine $m / z$ (the B-field is fixed). A high-voltage op-amp (Apex PA88) drives a voltage-divider network, from which are taken individual plate potentials (that determine the Efield, as shown in Fig. 3) and ion acceleration voltage. The DC voltage is controlled by computer, via an electrically isolated AD 569 digitalto-analog converter. Ionization is by electron impact $(70 \mathrm{eV})$, using a high-efficiency emission regulator based on a TL494 switching power supply controller chip and a pair of IFR530 MOSFETS, providing filament current at ca. 80 $\mathrm{kHz}$ through a custom-wound transformer (Pico \#10929). An optically isolated trap current signal is used to control the duty cycle of the TL494, providing feedback control of electron current while maintaining high overall circuit efficiency. Electron acceleration potentials are provided by miniature, modular DC-DC converters (Pico 'A' series).

The analyzer is operated in a B-field of approximately 0.42 Tesla provided by a magnet using high energy-product $\mathrm{Nd}-\mathrm{Fe}-\mathrm{B}$ material, similar to that described by [20], except that the magnetic material has an increased energy product and the flux path has been aggressively trimmed at points of low flux density to decrease weight. It is noteworthy that, using $\mathrm{Nd}-\mathrm{Fe}-\mathrm{B}$ permanent magnet material, total magnet weight often becomes dominated by the need to provide a non-saturated flux return path, rather than by the need for sufficient coercive force to establish the desired B-field in the air gap, as may be the case with older types of permanent magnet materials.

Ion current is detected with a Faraday cup, and an electrometer based on an LMC6001 opamp connected as a current-to-voltage converter. A $10^{11} \Omega$ feedback resistor is used.

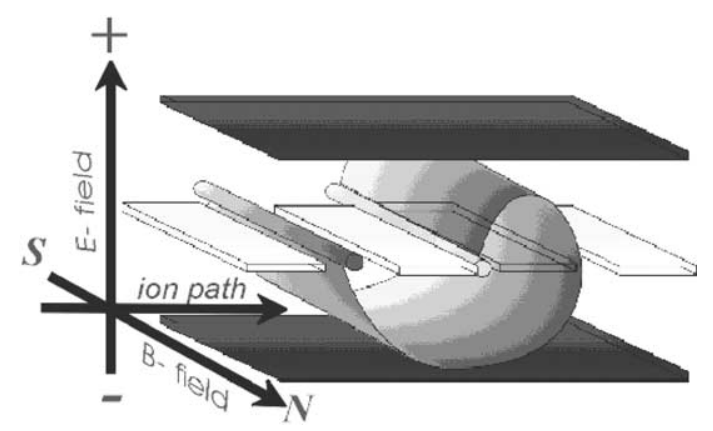

Fig. 3. Ion trajectory of a cycloidal focusing mass analyzer

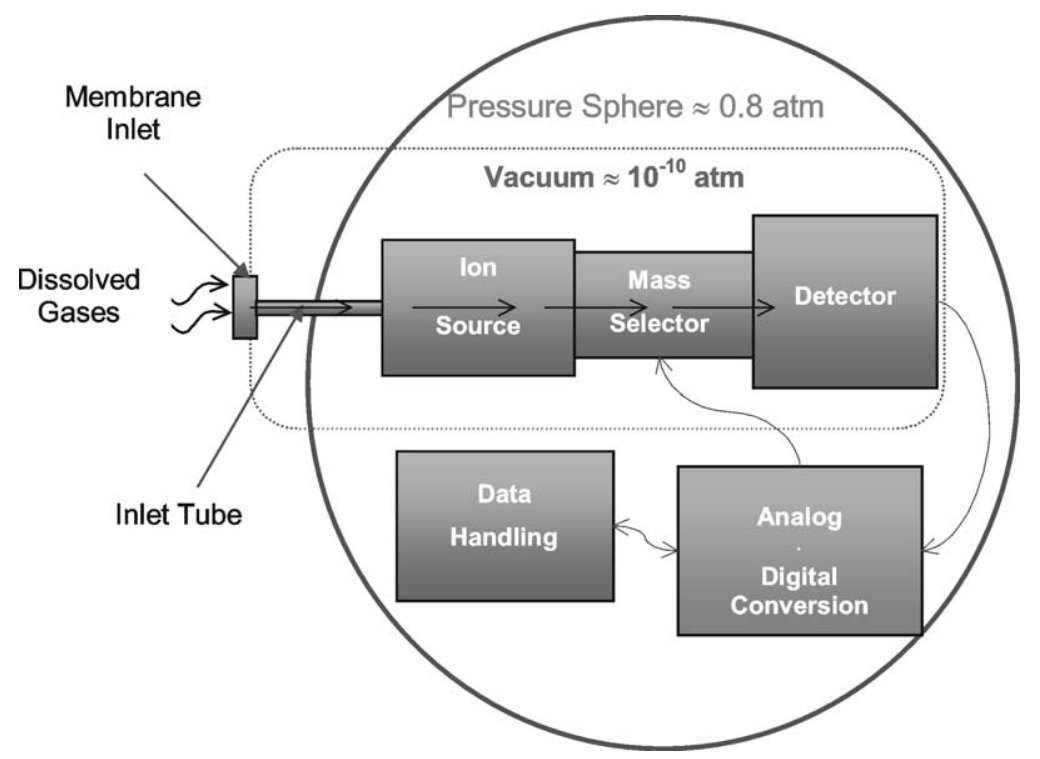

Fig. 2. NEREUS configuration showing flow of analyte and data. High vacuum is provided by an ion pump (not shown). 
Electrometer output is buffered and digitized using an Analog Devices 7884 (16-bit) A/D converter. A 386-based PC-104 embedded computer running at $25 \mathrm{MHz}$ interprets and stores data while contributing a power drain of less than 5 watts.

Autonomous functions now written or being written into the software for NEREUS include:

- powering various instrument components on and off at predetermined intervals (or in response to state conditions) to save energy;

- performing mass calibration;

- controlling $\mathrm{m} / \mathrm{z}$ scanning;

- collecting and processing data; and,

- responding to emergencies such as vacuum system failure.

In the future, spectrum separation will be accomplished in near-real time using the UNMIX algorithm [23]. When used on an Odyssey-class AUV, the PC-104 computer will use a serial line to communicate with the AUVcontrol computers to assist in those functions that are related to vehicle location and status. Alternatively, a wireless transceiver will allow an operator to communicate with NEREUS when it is on the surface, and accomplish data download, calibration checks or new mission upload.

\subsection{High-vacuum system}

Maintenance of high vacuum is a significant challenge for an underwater instrument that must operate on limited power. Gas and vapor flow through the membrane inlet includes a significant amount of water vapor as well as analyte. Roughing pump-turbopump combinations were deemed to have unacceptably high power consumption for the intended missions of NEREUS; thus we have used an $8 \mathrm{l} / \mathrm{s}$ diode ion pump (Varian) to maintain high vacuum in the analyzer. 3000 volts DC at up to several hundred $\mu \mathrm{A}$ are supplied to the ion pump by a DC-DC converter (Gamma High Voltage Research). Similarly to the analytical magnet, the magnetic circuit of the magnet has been aggressively trimmed in regions of low flux density, resulting in lightening by approximately $1.5 \mathrm{~kg}$ with no decrease in the 0.12 Tesla flux density. With this system, initial vacuum in the instrument must be obtained with an external roughing pump. After high vacuum is attained, the ion pump remains energized continuously.

\subsection{Membrane inlet}

The membrane inlet of NEREUS represents a trade-off among sensitivity to low concentrations of analyte, time response, ability to withstand external water pressure, and ease of construction. Because only a limited pressure differential can be withstood by the small-diameter dimethylsilicone tubing commonly used as a membrane inlet, we designed the inlet in a flat-plate configuration (Fig. 4) using a sheet supported by a porous support plate. The membrane and the plate are held in position using a PTFE washer and stainless steel retaining ring, which is threaded onto the inlet body. The porous plate is made of 304 stainless steel aperture mask material, $0.08 \mathrm{~cm}$ in thickness and approximately $0.63 \mathrm{~cm}$ in radius, with a porosity of approximately $12 \%$. The porous plate is currently the depth-limiting component. The inlet can be designed to withstand arbitrarily high pressure by making the plate as thick as necessary and the pores as small as necessary. The degree to which operation at very large depths (pressures) will require corrections to instrument calibration is not yet known.

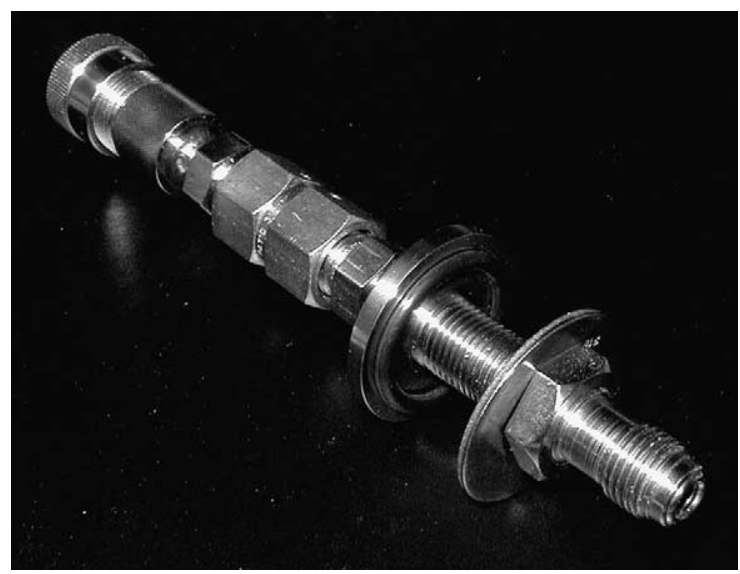

Fig. 4. Inlet apparatus used by the NEREUS instrument. 


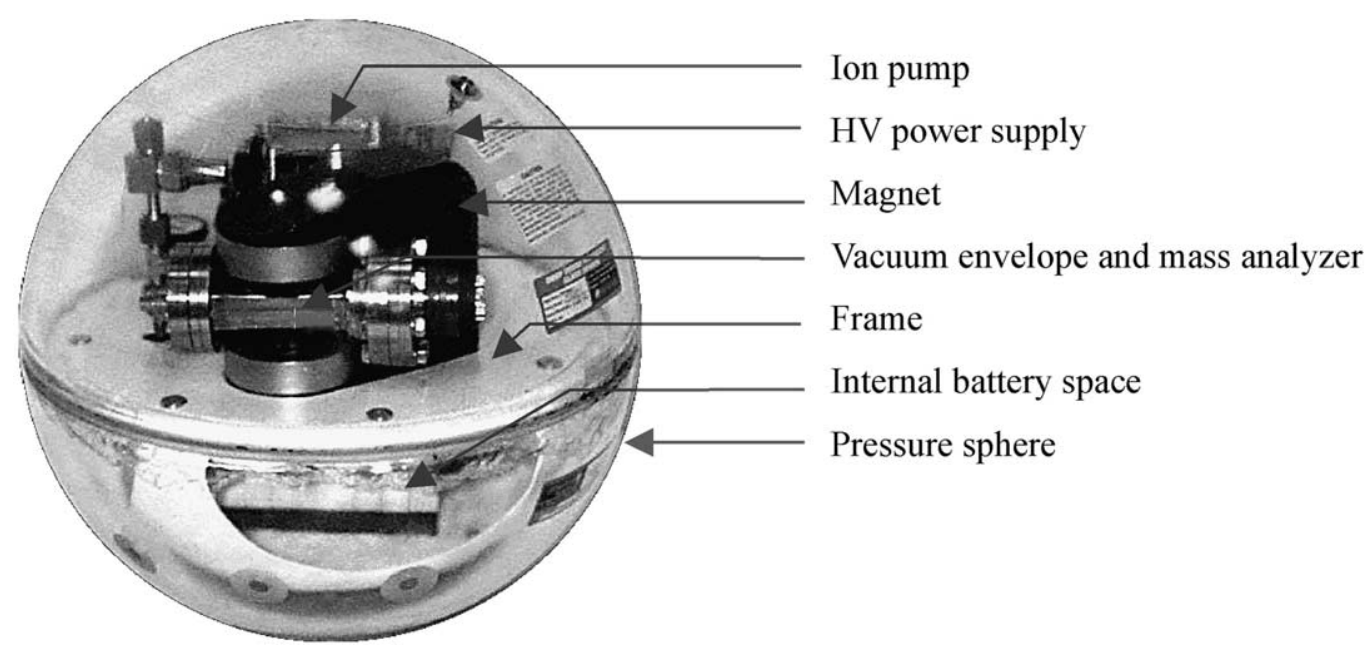

Fig. 5. Physical arrangement of major components of NEREUS within the glass pressure sphere. Electronics and computer are mounted independently in the volumes between the major components in the upper hemisphere.

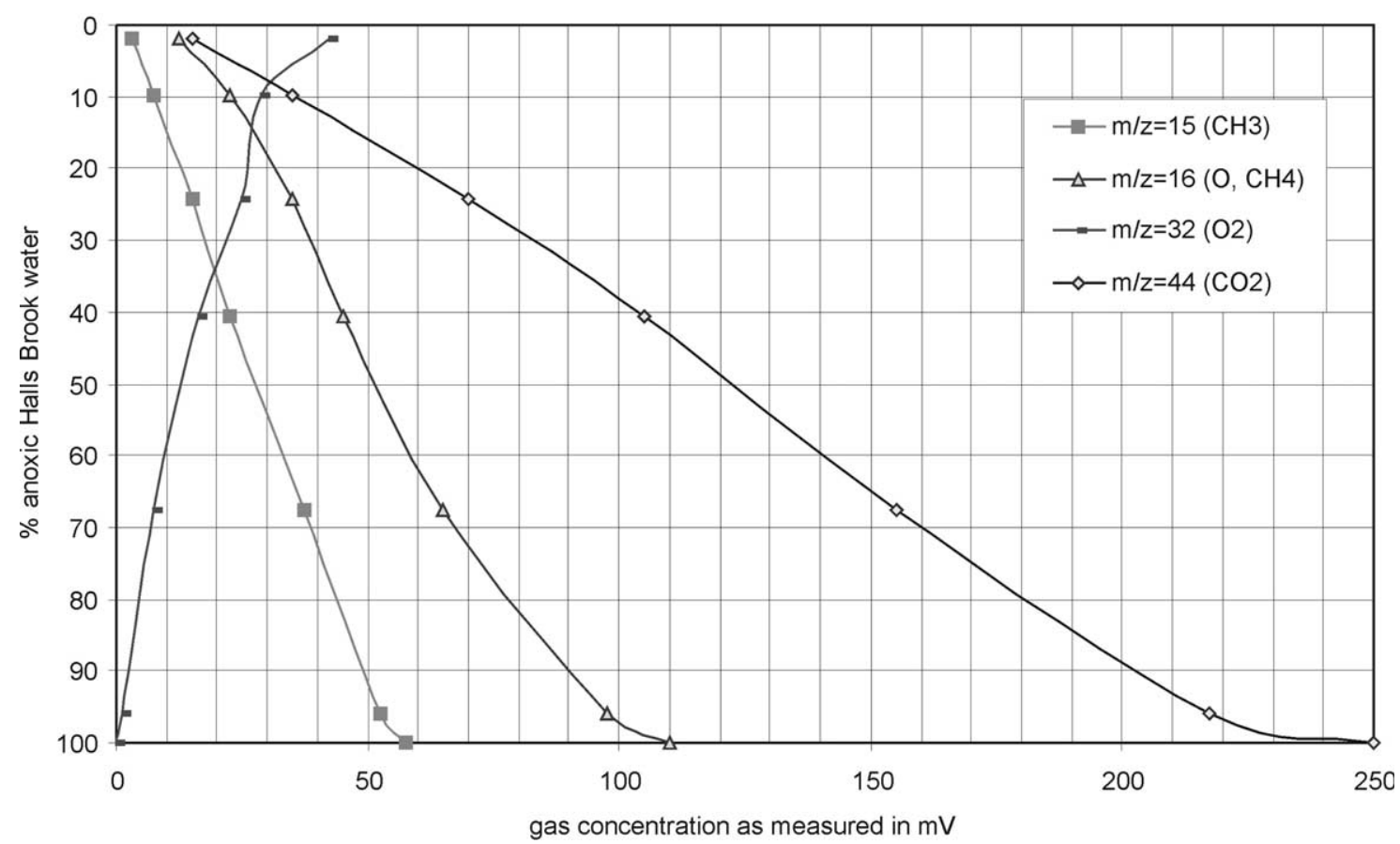

Fig. 6. Measurements of dissolved gases in Mishawum Lake water. The vertical gradients in this plot are created by mixing water collected from the anoxic, methanic monimolimnion of the lake with oxic tap water in varying proportions, and contacting the resulting waters with the membrane inlet of NEREUS. Oxygen concentration of the oxic end-member approximates 10 ppm.

\subsection{Mechanical lay-out}

The NEREUS prototype, mounted within its pressure sphere, is shown in Fig. 5. Because significant compression of the sphere occurs during deep submergence, these components are held in place using a composite support frame and mounting ring cast into the lower hemisphere. Space within the lower hemisphere is reserved for batteries. There is room for a 
24 amp-hour lead-acid battery that will provide 12 hours of continuous operation; this can be extended greatly by use of alternative battery chemistries. The membrane inlet is outside the hull of the AUV, and connection to the analyzer is accomplished via a 6-mm-diameter stainlesssteel inlet tube, traversing the lower hemisphere via a custom penetration fitting. Overall, the NEREUS instrument package is neutrally buoyant in sea water, and has a mass of less than $25 \mathrm{~kg}$ (including batteries).

\section{Experimental results}

A simulated spatial gradient of several gases at in-situ concentrations, as measured by NEREUS, is shown in Fig. 6. Bottom water known to be anoxic and to contain methane [24] was collected for this experiment in a BOD (biochemical oxygen demand) bottle from the Mishawum Lake (also known as Halls Brook Storage Area) in the Aberjona River watershed, north of Boston, MA, USA. Intermediate points in Fig. 6 were created by mixing samples of this water in appropriate ratios with "Q" water that simulated surface water having dissolved gas content near atmospheric equilibrium $(\sim 10$ ppm $\left.\mathrm{O}_{2}\right)$. The mixtures were contacted with NEREUS's membrane inlet and scans initiated. Results show successful simultaneous measurement of multiple gases, with high signalto-noise ratios, at useful and representative environmental concentrations.

\section{Discussion}

While the feasibility of mass spectrometry for in-situ underwater measurements of volatile solutes has now been clearly demonstrated, the full potential of the technique has not yet been realized, partly because underwater MSs are still individually custom-built and not readily available to a large group of potential users. In addition, technical improvements are still needed, especially in depth capability and power consumption. For certain purposes, additional sensitivity, more rapid time response, and expanded mass range are also needed. Rapid progress may be expected in some of these areas, while others are more problematic.

It is clear that greatly increased depth capability can be obtained. By contrast, decreasing power consumption significantly below that of NEREUS may be more difficult. The major reason is that the hot filament (used for electron emission for electron impact ionization) consumes almost half the power budget. Because this amount of power is dictated by heat-transfer physics, it is difficult to reduce greatly while using a hot filament. Alternative designs using cold electron sources may be necessary.

Increased sensitivity would make it possible to measure additional trace gases, such as hydrogen or nitrous oxide, the concentrations of which are diagnostic of geochemical processing in an ecosystem. Additional sensitivity would also allow smaller pollution sources to be detected at greater distances. In the case of NEREUS, improved electrometer design will help to realize this goal. Alternatively, other analyzer designs using an electron-multiplier detector can take advantage of the large amount of nearly noise-free gain such devices can provide to achieve higher sensitivity than is available from a Faraday-cup detector. Also, improved vacuum pumps would be highly desirable, and would lead to increased sensitivity by allowing more analyte inflow.

A variety of mass-analyzer designs can provide increased mass range to an underwater MS. However, a limiting factor in the case of membrane-inlet MSs is likely to be membrane permeability, which may become so low for higher molecular weight chemical species (say, larger than 100-200 Daltons) that alternative sample introduction techniques will have to be introduced. The possibilities opened by the use of electrospray may help overcome this limitation.

\section{Acknowledgements}

This research has been supported by the MIT Sea Grant College Program, The National Science Foundation, the William E. Leonhard Professorship and the Alfred E. Sloan Foundation. 


\section{References}

[1] I. Bussman, E. Suess, Continental Shelf Res. 18 (1998) 1795.

[2] J. Alper, Am. Soc. Microbiol. News 56 (1990) 536.

[3] T.S. Bates, K.C. Kelly, J.E. Johnson, R.H. Gammon, J. Geophys. Res. 101 (1996) 6953.

[4] J.I. Ishibashi, H. Wakita, Y. Nojiri, D. Grimaud, P. Jeanbaptiste, T. Gamo, J.M. Auzende, T. Urabe, Earth Planet. Sci. Lett. 128 (1994) 183.

[5] T. Takahashi, C. Wunsch, et al., Applications of Analytical Chemistry to Oceanic Carbon Cycle Studies, in Oceanographic Measurements; Technologies for Chemical Measurements, National Academy of Sciences, Washington DC, USA, 1993, p. 85.

[6] J.E. Johnson, Anal. Chim. Acta 395 (1999) 119.

[7] S. Park, Ocean Res. 17 (1995) 59.

[8] B.J. Harland, P.D. Nicholson, E. Gillings, Water Res. 21 (1987) 107.

[9] G. Hoch, B. Kok, Arch. Biochem. Biophys. 101 (1993) 160.

[10] T. Kotiaho, J. Mass Spectrom. 31 (1995) 1.

[11] S. Richardson, Chem. Rev. 101 (2001) 211.

[12] A. Maden, M. Hayward, Anal. Chem. 68 (1996) 1805.

[13] G. Baykut, J. Franzen, Trends Anal. Chem. 13 (1994) 267.

[14] W. McDonald, M. Erickson, B. Abraham, A. Robbat, Environ. Sci. Technol. 28 (1994) 336A.

[15] J. Evans, J Arnold, Environ. Sci. Technol. 9 (1975) 1134.

[16] E. Badman, R. Cooks, J. Mass Spectrom. 35 (2000) 659.

[17] R.T. Short, et al., J. Am. Soc. Mass Spectrom. 12 (2001) 676.

[18] G. McMurty, S. Smith, Mass SURFER field mass spec- trometer system for deep ocean and planetary lander applications, Third Workshop on Harsh-Environment Mass Spectrometry, Pasadena, CA, USA, 25-28 March 2002.

[19] G. Matz, W. Schroder, M. Muller, G. Kibelka, Underwater MI/GC/MS, unpublished brochure, Technical University of Hamburg-Harburg, ca. 2001.

[20] H.F. Hemond, Rev. Sci. Instrum. 62 (1991) 1420.

[21] C.F. Robinson, L.G. Hall, Rev. Sci. Instrum. 27 (1956) 504.

[22] W. Bleakney, J. Hipple, Phys. Rev. 53 (1938) 521.

[23] R. Doherty, An Algorithm for Mixture. Component Determination for use in Membrane Inlet Mass Spectrometry, M.S. thesis, Department of Civil and Environmental Engineering, Massachusetts Institute of Technology, Cambridge, USA, 1987.

[24] L. Wick, K. McNeill, M. Rojo, E. Medilanski, P.M. Gschwend, Environ. Sci. Technol. 34 (2000) 4354.

Harry Hemond is W. E. Leonhard Professor of Civil and Environmental Engineering, and Director of MIT's R. M. Parsons Laboratory. He is a biogeochemist, with research interests that include arsenic cycling, and the use of metabolic gases as indicators of biogeochemical transformations in ecosystems. Hemond is also author, with E. Fechner-Levy, of the textbook Chemical Fate and Transport in the Environment.

Richard Camilli is a doctoral student at the R. M. Parsons Laboratory at MIT. His research interests include instrumentation, and intelligent control of autonomous environmental sensors. 